\section{The Role of the Frontal Cortex in Task Preparation}

Marcel Brass and D. Yves von Cramon

Max Planck Institute of Cognitive Neuroscience, Leipzig, Germany

\begin{abstract}
The ability to prepare a task is crucial for the voluntary control of our actions. It enables us to react flexibly and rapidly to a changing environment. In the present event-related functional magnetic resonance imaging study we investigated task preparation with a task-cueing paradigm. In this paradigm we intermixed trials in which a task cue and a target were presented with trials in which only the task cue was presented. Analysis of these cue-only trials allowed us to isolate task-preparation related control from execution-related control processes. By means of this paradigm, we could demonstrate that a frontal network was related to task preparation. Further analysis revealed that the fronto-lateral cortex at the junction of precentral sulcus and inferior frontal sulcus and the presupplementary motor area are the crucial frontal components in task preparation.
\end{abstract}

\section{Introduction}

One central question of cognitive neuroscience is how we are able to voluntarily control our thoughts and actions. The ability to prepare a task is crucial in this respect (Monsell, 1996). Task preparation allows us to react flexibly and rapidly to environmental events and to plan actions in advance to concrete environmental conditions. In cognitive psychology the process of task preparation was investigated using task cueing paradigms (Sudevan and Taylor, 1987; Meiran, 1996). In such paradigms subjects usually have to switch between two tasks. In some trials a task cue is presented in advance of the task, allowing preparation for the task. It was found that knowing in advance which task to execute leads to a reduction in reaction time (Sudevan and Taylor, 1987). This benefit was interpreted as a measure of task preparation. However, it has been established that we are not able to completely prepare the task in advance (Meiran, 1996). Some adjustments can only take place when the environmental conditions for task execution are specified exactly (Allport et al., 1994; Rogers and Monsell, 1995; Meiran, 1996). Therefore, the behavioral results suggest that task-related control processes can be decomposed into two components: a preparation-related component and a target-related component. Only the first component was assumed to reflect endogenous task-management processes (Rogers and Monsell, 1995). Consequently, it is necessary to isolate this component, in order to make strong conclusions about the neural mechanisms underlying task management.

Paradigms in which subjects have to switch between different tasks are suitable to investigate task preparation, since the need to prepare the task in each trial is high. Recent neuroimaging studies have revealed the cortical mechanism of task-related control processes in general. To switch from one task to another is related to activation of the fronto-lateral cortex, the frontomedian cortex and parietal cortical areas (Konishi et al., 1998; Omori et al., 1999; Dove et al., 2000; Kimberg et al., 2000; Pollmann et al., 2000). However, the fundamental question of whether task preparation involves different brain regions than target-related control was not addressed in these studies.

Only two studies tried to separate preparation-related activation from target-related activation in a task-switching paradigm, using brain imaging techniques (MacDonald et al., 2000; Sohn et al., 2000). The study of Sohn and colleagues suggested that the ventro-lateral prefrontal cortex and the superior parietal cortex is related to task preparation. The study of MacDonald and colleagues has found preparation-related activation in the middle frontal gyrus (BA 9). Both studies tried to separate preparationrelated activation from execution-related activation by using a long preparation interval. In the study of MacDonald and colleagues the delay was $12.5 \mathrm{~s}$, and in the study of Sohn and colleagues it was $6 \mathrm{~s}$. Since it is known from behavioral taskswitching studies that the optimal preparation interval is a few hundred milliseconds (Meiran, 1996), these long preparation intervals might have included working memory processes in addition to task preparation. Therefore, the aim of the present study was to investigate task preparation by using a paradigm with a short cue-target interval that nevertheless allows for a clear separation of preparation-related control.

Subjects were instructed to switch between two tasks. In some trials, a task cue was given in advance to the target to instruct subjects which task was to be executed. This task cue was followed by a target to which subjects had to respond. In some trials the task instruction and target were presented concurrently. Importantly, sometimes only the task cue was presented but not the target. By looking at cue-only trials, we were able to isolate preparation-related activation from targetrelated activation without using a long cue-target interval.

\section{Materials and Methods}

\section{Subjects}

Twelve healthy volunteers were recruited. We obtained written consent from all 12 subjects prior to the scanning session. All subjects had normal or corrected-to-normal vision. No subject had a history of neurological, major medical, or psychiatric disorder. One subject's data were excluded from the analysis, due to significant head motion. The remaining 11 subjects were six males and five females (age: mean $=26.2, \mathrm{SD}=3.06$ ) who were all right-handed as assessed by the Edinburgh Inventory (Oldfield, 1971).

\section{Behavioral Task}

Digits between 20 and 40 (except 30) were presented on the computer screen. Participants had to execute two tasks: judging whether a digit was smaller or greater than 30 and judging whether the digit was odd or even. Which task they had to execute was instructed by the shape of a frame surrounding the digit (Fig. 1). A square indicated that the greater/smaller task should be executed and a diamond indicated that the odd/even task should be executed. Each trial started with a fixation cross that remained on the screen for $\sim 200 \mathrm{~ms}$. The task instruction (square or diamond) was presented either $1200 \mathrm{~ms}$ before the target (cue-target condition) or simultaneously with the target (no-cue-target condition). Subjects 


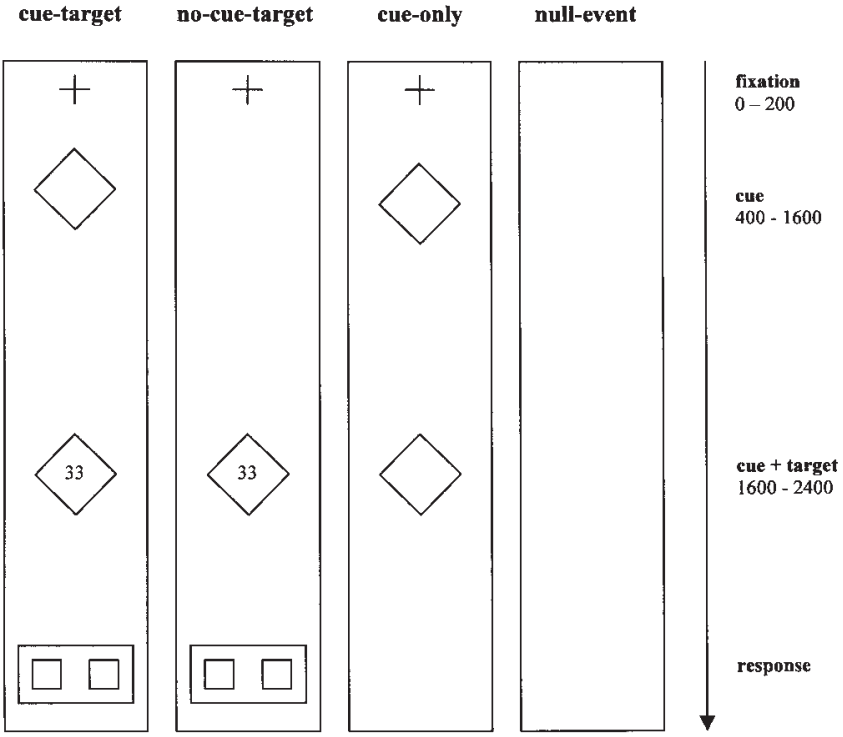

Figure 1. Digits between 20 and 40 were presented on the computer screen. Subjects had to execute two tasks: judging whether a digit was odd or even, or judging whether the digit was greater or smaller than 30. A frame surrounding the digit instructed the subject which task to execute. In cue-target trials, this frame was presented $1200 \mathrm{~ms}$ before the target appeared. In no-cue-target trials, the instructional cue was presented simultaneously with the target. In cue-only trials, only the cue was presented. Null events, in which a blank screen was presented, served as a baseline.

responded with a left key press to a digit that was smaller than 30 or odd, and with a right key press to a digit that was greater than 30 or even. In some trials, only the cue appeared but no target (cue-only condition) and remained on the screen for $2000 \mathrm{~ms}$. In addition, null events were presented in which neither a target nor a cue were presented. The whole experiment consisted of 388 experimental trials and 48 null events. Trials were presented in a pseudo-randomized order to equal the number of switch and repetition trials and the transition probabilities of the different conditions. The 388 experimental trials consisted of a three-way even split (128 trials) between cue-only trials, cue-target trials and no-cue-target trials.

\section{Magnetic Resonance Imaging (MRI) Scanning Procedure}

The experiment was carried out on a $3 \mathrm{~T}$ scanner (Medspec 30/100, Bruker, Ettlingen, Germany). Sixteen axial slices (19.2 cm FOV, $64 \times 64$ matrix, $5 \mathrm{~mm}$ thickness, $2 \mathrm{~mm}$ spacing), parallel to the AC-PC plane and covering the whole brain were acquired using a single-shot, gradient recalled EPI sequence $\left(T_{\mathrm{R}}=2000 \mathrm{~ms}, T_{\mathrm{E}}=30 \mathrm{~ms}, 90 \mathrm{flip}\right.$ angle $)$. Two functional runs with 445 time points each were run, with each time point sampling over the 16 slices. Prior to the functional runs, corresponding 16 anatomical MDEFT slices and $16 \mathrm{EPI}-T_{1}$ slices were acquired.

Stimuli were displayed by a LCD projector on a back-projection screen and mounted in the bore of the magnet behind the participants' head. Participants viewed the screen wearing mirror glasses. We used jittering and oversampling to increase the temporal resolution of the measurement (Miezin et al., 2000). Each trial had a trial length of $5 \mathrm{~s}$. Since the $T_{\mathrm{R}}$ was $2 \mathrm{~s}$, every second trial started in the middle of the image acquisition. In order to increase the temporal resolution to $500 \mathrm{~ms}$, we delayed half of the trials by $500 \mathrm{~ms}$ relative to the image acquisition. The experiment was carried out in two blocks. Each block consisted of 550 time steps and started with two practice trials that were excluded from further analysis.

\section{Data Analysis}

Preprocessing of Functional Magnetic Resonance Imaging (fMRI) Data Analysis of fMRI data was performed using the LIPSIA software package (Lohmann et al., 2001). First, functional data were corrected for movement artifacts. Then the temporal offset between the slices acquired in one scan were corrected using a sinc interpolation algorithm. Data were filtered using a spatial Gaussian filter with sigma $=1$. A temporal highpass filter with a cutoff frequency of $1 / 48 \mathrm{~Hz}$ was used for baseline correction of the signal. The increased autocorrelation that was due to filtering was taken into account during statistical evaluation.

All functional data sets were individually registered into 3D space using the subjects' individual high-resolution anatomical images. The 2D anatomical MDEFT slices, geometrically aligned with the functional slices, were used to compute a transformation matrix, containing rotational and translational parameters, that registers the anatomical slices with the $3 \mathrm{D}$ reference $T_{1}$ data set. This $3 \mathrm{D}$ reference data set was acquired for each subject during a previous scanning session. Geometrical distortions of the EPI- $T_{1}$ images were corrected using additional EPI- $T_{1}$ refinement on the transformation matrices. These transformation matrices were normalized to the standard Talairach brain size (Talairach and Tournoux, 1988) by linear scaling, and then finally applied to the individual functional data.

\section{Statistical Evaluation}

The statistical evaluation was carried out using the General Linear Model for serially autocorrelated observations (Friston et al., 1995). For each individual subject, statistical parametric maps (SPM) were generated. The design matrix for event-related analysis was created using a model of the hemodynamic response with a variable delay and its temporal derivative (Friston et al., 1998). The model equation was convolved with a Gaussian kernel with a dispersion of $4 \mathrm{~s}$ FWHM. The increased temporal autocorrelation caused by filtering was corrected by an adjustment of the degrees of freedom. The contrasts between the different conditions were calculated using $t$ statistics, and afterwards $t$ values were transformed to $z$ scores. Two different types of contrasts were computed: contrasts of the activation strength between two conditions, and contrasts of the time delay between two conditions. Group activation was calculated by one-sample $t$-test at corresponding voxels of individual $\operatorname{SPM}\{z\}$ across subjects (Bosch, 2000). Resulting $\operatorname{SPM}\{z\}$ was thresholded at $z>6.1$ for the contrasts of activation strength and at $z>3.1$ for the difference in temporal delay between experimental conditions. In order to be able to isolate only the most crucial activation foci we have chosen a relatively high $z$ threshold $(z>6.1)$ for the activation contrasts which yielded very high $z$ values. Since we restricted the analysis of time delay differences to the frontal cortex, we think the lower $z$ threshold of 3.1 is justified. All $z$ maps were mapped onto a mean brain, averaged out of the individual high-resolution anatomical data sets of the 11 subjects which have been normalized by non-linear scaling (Thirion, 1998).

The correlational analysis was computed by correlating the percent signal change of each subject in the relevant brain region with the behavioral cueing effect in milliseconds. First we computed the reaction time difference in cue-target trials and no-cue-target trials. Then the percent signal change was computed for each subject and each brain region. The average time course of the bold response over 27 voxels for each condition was computed at the location of the $z_{\max }$ in the mean $z$ map of cue-only trials. Then the time course of the null events was subtracted from each condition. Finally, the percent signal change was computed by subtracting the first timestep from the maximal signal change value within a given time-window (3-8 s).

The analysis of the temporal delay of the bold response was computed by contrasting the temporal derivative of the basis function (Friston et al., 1998). The comparison of temporal delay of fast and slow responses was assessed by dividing the RT distribution of each subject in quintiles (Ratcliff, 1979). The temporal delay of trials from the first and fifth quintile were compared.

\section{Results}

\section{Behavioral Results}

The behavioral results revealed a significant main effect for cueing, $F(1,10)=74,11, P<0.01$ (Fig. 2 ). If subjects received a cue in advance of the target they reacted $221 \mathrm{~ms}$ faster compared with the condition where cue and target were presented simultaneously. In addition, a task-switching effect of $40 \mathrm{~ms}$ was found, $F(1,10)=9.01, P<0.05$. 


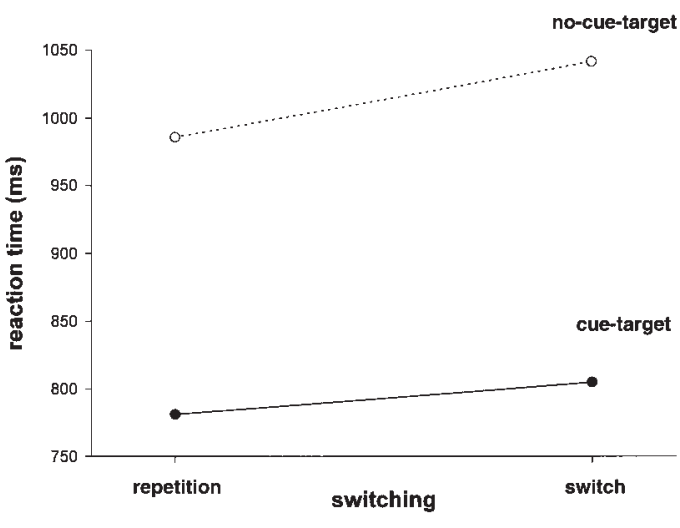

Figure 2. Reaction time as a function of cueing (cue vs no-cue) and switching (switch vs repetition).

\section{Neuroimaging Data}

The first question we addressed in the analysis of the fMRI data was whether the mere presentation of a cue led to activation in the frontal cortex. The comparison of cue-only trials with null events revealed fronto-lateral activation at the junction of the precentral sulcus and inferior frontal sulcus (inferior frontal junction, IFJ) in both hemispheres (Fig. $3 A$ ). In addition, activation was found in the middle frontal gyrus (MFG) and the dorsal premotor cortex, bilaterally. The insula was activated in the right hemisphere. Medially, activation was found in the pre-supplementary motor area (pre-SMA). Furthermore, the parietal lobe was found to be activated along the intraparietal sulcus (IPS) and the upper precuneus. Finally, some activation foci were found in the occipital cortex and are not discussed in the present paper (Table 1). The contrast of cue-target trials and cue-only trials (Fig. 3B), which reflects target-related processing, revealed frontal activation in different regions of the premotor cortex and the hand field of the motor cortex. In addition, an activation was found in the left inferior frontal junction (IFJ). Medially, activation was found in the anterior cingulate cortex (ACC; Table 2).

The same frontal foci as in the cue-only versus null event contrast were found in the null-event contrasts of cue-target trials (see Table 3 for a listing of the frontal activation).

In order to investigate whether the cortical activation in the frontal cortex was related to task preparation we computed the correlation of activation strength in the cue-only condition and the behavioral cueing effect for all activated frontal areas in the cue-only condition. A significant positive correlation was only found for the left IFJ activation $(r=0.63, P<0.05)$ and the pre-SMA $(r=0.57, P<0.05)$ (Fig. $3 A$ ). The right IFJ was not correlated with the cueing effect, $r=-0.07, P=0.82$.

The second part of the analysis was related to the question of whether the frontal activation, which was found in cue-only trials, differed between cue-target trials and no-cue-target trials. If the frontal activation is indeed relevant for task preparation, as suggested by the first part of the analysis, one would expect differences in cue-target trials compared to no-cue-target trials.
Table 1

Anatomical location, Talairach coordinates and maximum $z$ values for the contrast of cue-only trials and null events. Activated areas that lie $>1 \mathrm{~cm}$ apart and have a $z$ value $>6.1$ are reported

\begin{tabular}{|c|c|c|c|c|}
\hline \multirow[t]{2}{*}{ Anatomical area } & \multicolumn{2}{|c|}{ Left hemisphere } & \multicolumn{2}{|c|}{ Right hemisphere } \\
\hline & $\begin{array}{l}\text { Talairach } \\
\text { coordinates }\end{array}$ & $z_{\max }$ & $\begin{array}{l}\text { Talairach } \\
\text { coordinates }\end{array}$ & $Z_{\max }$ \\
\hline \multicolumn{5}{|l|}{ Frontal } \\
\hline IFJ (BA 6/8/44) & $-40,-1,32$ & 10.782 & $44,-1,38$ & 10.907 \\
\hline Precentral gyrus (BA 6) & $-32,-7,44$ & 10.287 & $34,-4,44$ & 10.661 \\
\hline Middle frontal gyrus (BA 9) & $-34,32,32$ & 7.363 & $37,32,30$ & 11.256 \\
\hline Medial frontal gyrus (BA 6) & & & $1,5,53$ & 13.115 \\
\hline Insula/inferior frontal gyrus (BA 44) & & & $35,14,15$ & 8.25 \\
\hline \multicolumn{5}{|l|}{ Parietal } \\
\hline Intra-parietal sulcus (BA 7) & $-34,-46,47$ & 10.816 & $31,-55,44$ & 8.833 \\
\hline Superior parietal lobe (BA 7) & $-26,-67,38$ & 9.551 & & \\
\hline Precuneus (BA 7) & & & $8,-67,44$ & 8.806 \\
\hline Inferior parietal lobe (BA 40) & & & $59,-46,32$ & 7.215 \\
\hline Cingulate gyrus (BA 31) & & & $4,-34,24$ & 6.903 \\
\hline \multicolumn{5}{|l|}{ Temporal } \\
\hline Middle temporal gyrus (BA 21) & & & $55,-55,9$ & 8.408 \\
\hline \multicolumn{5}{|l|}{ Occipital } \\
\hline Fusiform gyrus (BA 19) & $-37,-64,-12$ & 14.26 & & \\
\hline Superior occipital gyrus (BA 19) & & & $34,-73,32$ & 7.753 \\
\hline Inferior occipital gyrus (BA 18) & $-31,-91,6$ & 20.337 & $37,-73,0$ & 14.841 \\
\hline Lingual gyrus (BA 18) & & & $8,-79,-12$ & 10.800 \\
\hline Calcarine sulcus (BA 17) & $-16,-97,6$ & 20.244 & $19,-94,6$ & 18.768 \\
\hline
\end{tabular}

Two testable predictions regarding these differences are conceivable. One prediction is that in trials in which a cue was presented before the target, an additional process of task preparation takes place. The alternative prediction assumes that task preparation is required no matter whether the task instruction is given in advance of the target or not. The first prediction assumes a difference in the activation strength between cue-target trials and no-cue-target trials. The second prediction assumes a temporal delay between these trial types. While the contrast of activation strength showed no significant frontal difference, some frontal brain areas showed a time delay in no-cue-target trials compared to cue-target trials as revealed by the contrast of the temporal derivative (Fig. 3C, note that this $z$ map displays the cortical areas which showed a significant time delay in these two trial types and not the significance of differences in the amplitude). A significant time delay was found for the IFJ, the dorsal premotor cortex, the pre-SMA, the frontoopercular cortex and the anterior inferior frontal sulcus (Table 4).

Finally, we wanted to investigate whether there was a relationship between the activation onset in the IFJ and the pre-SMA and the size of the behavioral cueing effect. One could assume that subjects did not always use the cue in cue-target trials. According to our logic, this should lead to a delay of the cue-related activation in the frontal cortex and long reaction times. We tested this assumption by contrasting trials with fast responses

Figure 3. (A) Contrast map of cue-only trials and null events which reflects cue-related activation. The activation was mapped on a mean brain of the 11 subjects and shows the activation $(z>6.1)$ in the IFJ (top), and the pre-SMA (bottom). The two scatter plots display the correlation of the cueing effect and the percent signal change in the IFJ and the pre-SMA. $(B)$ Contrast map of cue-target trials and cue-only trials which reflects target-related activation. The lateral view displays the primary motor activation. The medial view displays activation in the anterior cingulate gyrus. (C) A left view of the fronto-lateral cortex showing a significant $(z=3.1)$ time delay between cue-target and no-cue-target trials (Talairach coordinates of $\left.z_{\max }, x:-38, y: 2, z: 30\right)$. The picture beneath shows the time delay for the pre-SMA (x: 5, y: 11, z: 44). In addition, the time courses are displayed. The time course of the null event was subtracted from both conditions in order to adjust for the overlap of activation of previous trials. 


\section{A cue-only vs. null-event}
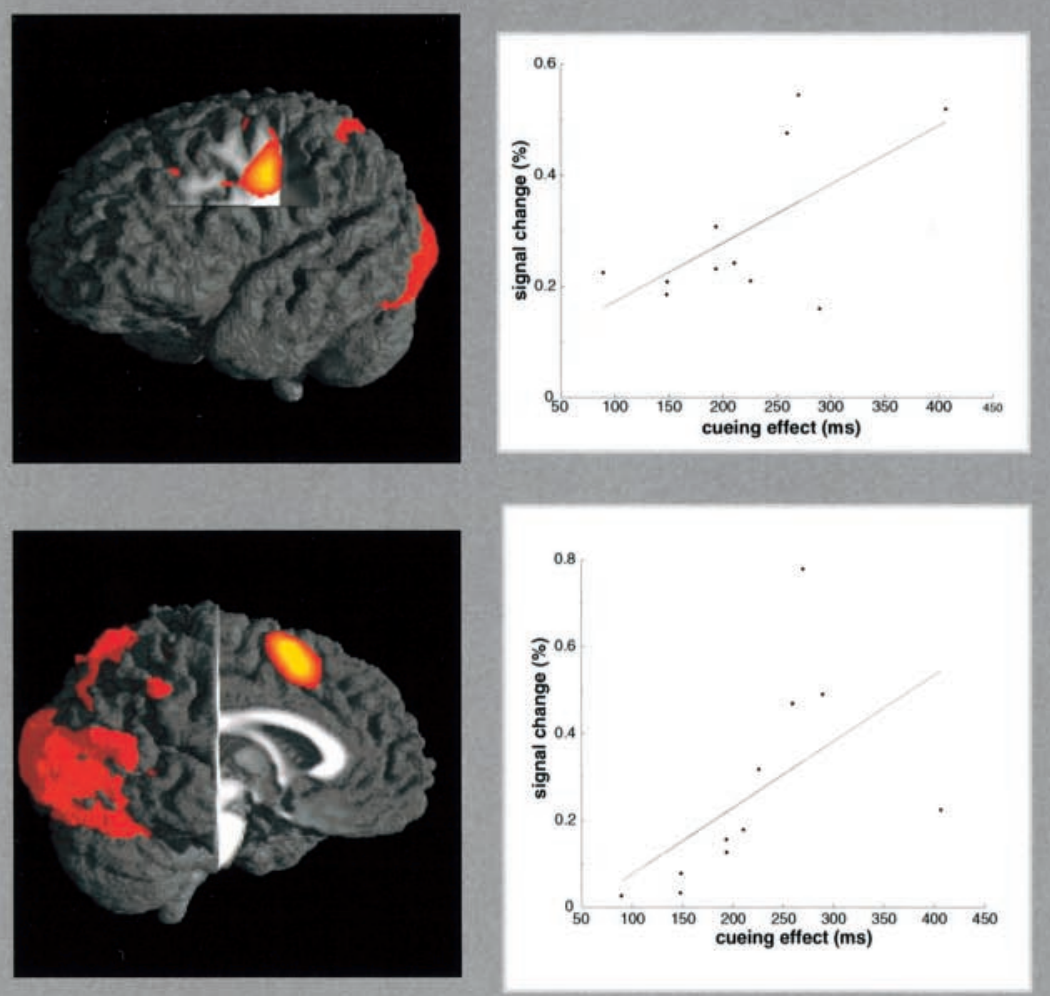

B cue-target vs. cue-only
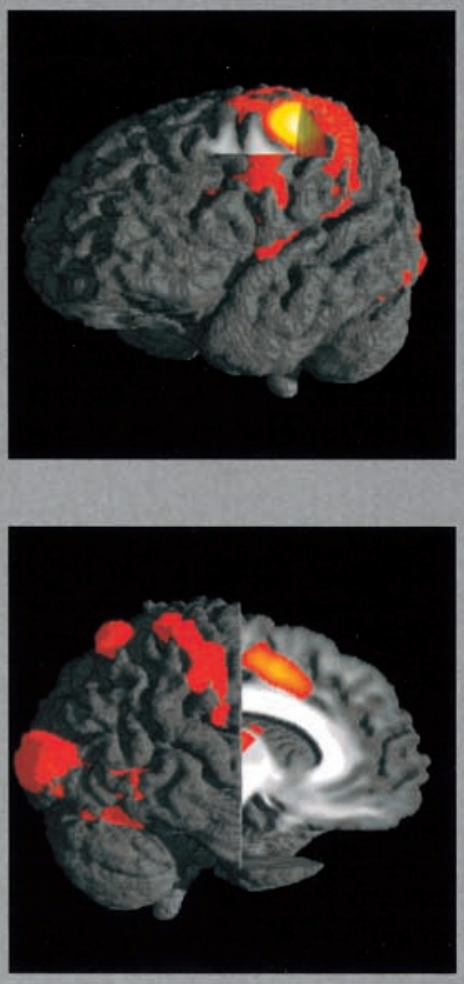

\section{C cue-target vs. nocue-target (time delay)}
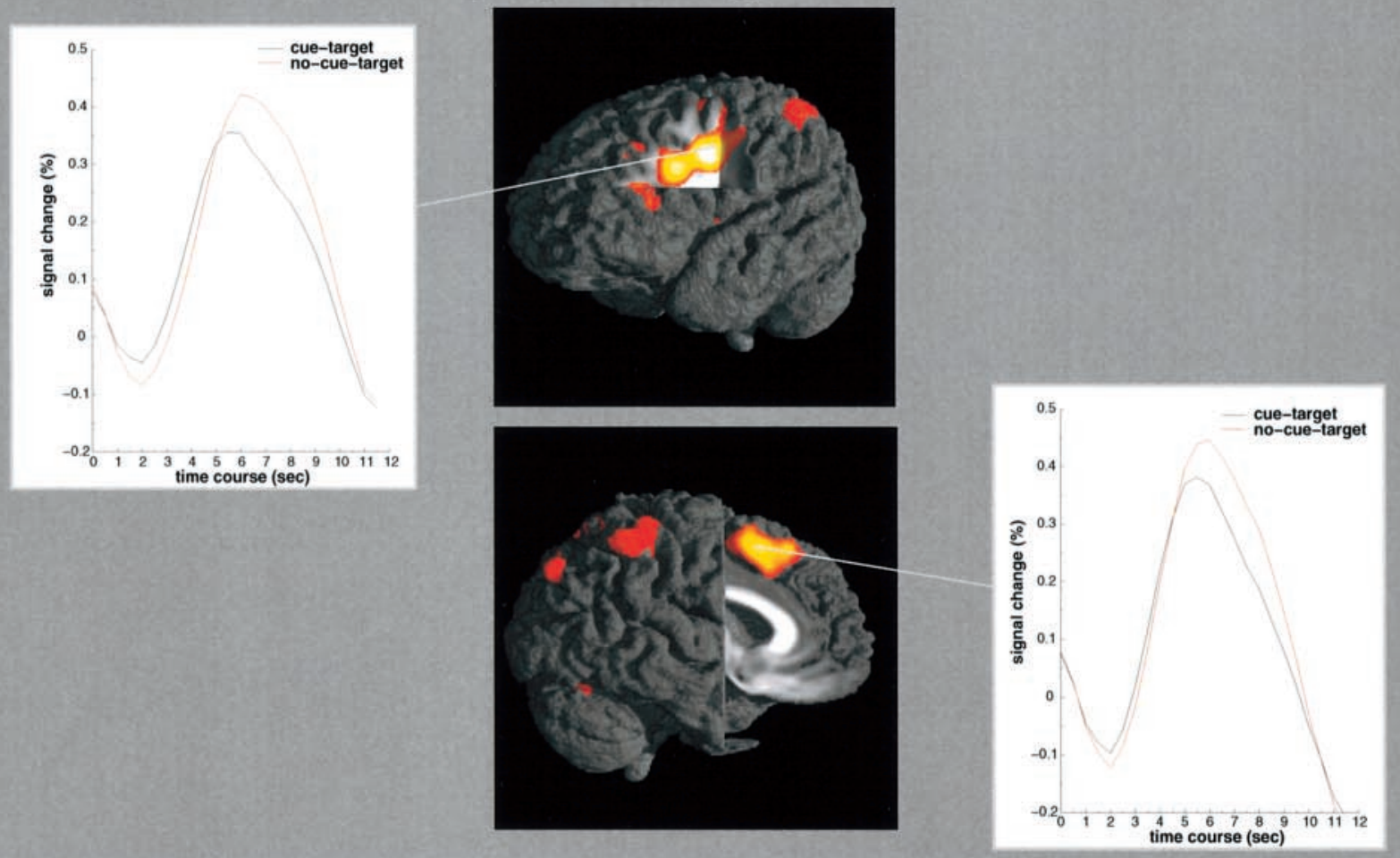
Table 2

Anatomical location, Talairach coordinates and maximum $z$ values for the contrast of cue-target trials and cue-only trials. This contrast reflects target-related processing. Activated areas that lie $>1 \mathrm{~cm}$ apart and have $\mathrm{z}$ value $>6.1$ are reported

\begin{tabular}{|c|c|c|c|c|}
\hline \multirow[t]{2}{*}{ Anatomical area } & \multicolumn{2}{|c|}{ Left hemisphere } & \multicolumn{2}{|c|}{ Right hemisphere } \\
\hline & $\begin{array}{l}\text { Talairach } \\
\text { coordinates }\end{array}$ & $Z_{\max }$ & $\begin{array}{l}\text { Talairach } \\
\text { coordinates }\end{array}$ & $z_{\max }$ \\
\hline \multicolumn{5}{|l|}{ Frontal } \\
\hline IFJ (BA 6/8) & $-38,2,32$ & 8.633 & & \\
\hline Precentral gyrus (BA 6) & & & $32,-10,59$ & 8.684 \\
\hline Precentral gyrus (BA 6) & $-52,2,27$ & 9.003 & $53,5,35$ & 8.770 \\
\hline Precentral gyrus (BA 4) & $-35,-22,50$ & 16.082 & & \\
\hline Anterior cingulate gyrus (BA 24) & $-4,2,44$ & 11.347 & & \\
\hline Insula & $37,-7,18$ & 9.112 & & \\
\hline \multicolumn{5}{|l|}{ Parietal } \\
\hline Postcentral gyrus (BA 40) & $-52,-22,24$ & 7.965 & $56,-28,35$ & 8.001 \\
\hline Postcentral gyrus (BA 43) & $-40,-10,18$ & 10.964 & & \\
\hline Intra-parietal sulcus (BA 7) & $37,-43,50$ & 7.183 & & \\
\hline Inferior parietal lobe (BA 7) & $34,-61,50$ & 8.036 & & \\
\hline Superior parietal lobe (BA 7) & $-26,-67,44$ & 7.508 & & \\
\hline \multicolumn{5}{|l|}{ Subcortical } \\
\hline Thalamus & $-11,-22,12$ & 9.914 & $10,-22,15$ & 8.223 \\
\hline Pulvinar & $-26,11,12$ & 8.010 & & \\
\hline \multicolumn{5}{|l|}{ Occipital } \\
\hline Gyrus lingualis (BA 19) & $23,-58,-9$ & 13.361 & & \\
\hline Inferior occipital gyrus (BA 18) & $32,-91,6$ & 13.198 & & \\
\hline Inferior occipital sulcus (BA 37) & $-38,-61,3$ & 9.142 & & \\
\hline Gyrus occipitalis medius (BA 18) & $-25,-97,0$ & 15.001 & & \\
\hline Calcarine sulcus (19) & $-23,-61,-9$ & 10.115 & & \\
\hline
\end{tabular}

(the first quintile of the RT distribution) with trials in which subjects reacted slowly (the fifth quintile of the RT distribution). A significant time delay $(P<0.005)$ was again found for the left IFJ $(x:-40, y:-1, z: 32)$ and the pre-SMA $(x: 1, y: 5, z: 53)$. No significant time delay $(P>0.01)$ was found for the primary motor cortex $(x: 35, y: 22, z: 50)$ as would have been expected if the difference reflected a simple delay of motor execution.

\section{Discussion}

The present study dissociated task-cue related from target-related processing in a task-switching paradigm. A frontal network including the IFJ, the dorsal premotor cortex, the frontal operculum and the pre-SMA was found to be related to the processing of the task cue. Target-related frontal activation was found in the ventral premotor cortex, the primary motor cortex and the ACC. Since the cue-related network was activated in trials in which only a cue but no target was presented, the results cannot be traced back to target-related control processes. Nevertheless, one might argue that cue-only trials involved additional cognitive processes caused by the non-appearance of the target. The analysis of cue-target trials contradicts this alternative explanation, since the same frontal areas were found to be involved. Another interpretation of the cue-related activation might be that it reflects general stimulus processing that is not related to task preparation. However, the correlation of the activation strength in cue-only trials and the size of the behavioral cueing effect indicated that the left IFJ and the pre-SMA are strongly related to task preparation. Surprisingly, the right IFJ was completely uncorrelated with the cueing effect, which suggests a less specific functional role in task preparation. At present, we can only speculate about the causes of this
Table 3

Anatomical location, Talairach coordinates and maximum $z$ values for the contrast of cue-target trials and null events. Activated areas in the frontal cortex that lie $>1 \mathrm{~cm}$ apart and a have $z$ value $>6.1$ are reported

\begin{tabular}{|c|c|c|c|c|}
\hline \multirow[t]{2}{*}{ Anatomical area } & \multicolumn{2}{|c|}{ Left hemisphere } & \multicolumn{2}{|c|}{ Right hemisphere } \\
\hline & $\begin{array}{l}\text { Talairach } \\
\text { coordinates }\end{array}$ & $z_{\max }$ & $\begin{array}{l}\text { Talairach } \\
\text { coordinates }\end{array}$ & $Z_{\max }$ \\
\hline \multicolumn{5}{|l|}{ Frontal } \\
\hline IFJ (BA 6/44) & $-40,2,32$ & 19.218 & $47,5,32$ & 17.715 \\
\hline Precentral gyrus (BA 6) & $-28,-7,50$ & 18.370 & $34,-4,47$ & 16.923 \\
\hline Middle frontal gyrus (BA 9) & $-34,32,32$ & 10.005 & $38,32,30$ & 14.256 \\
\hline Medial frontal gyrus (BA 6) & $-2,5,47$ & 23.277 & & \\
\hline Inferior frontal sulcus (BA 45/46) & & & $40,35,18$ & 10.286 \\
\hline Insula/inferior frontal gyrus (BA 44) & $-29,14,12$ & 15.224 & $32,14,12$ & 14.744 \\
\hline
\end{tabular}

Table 4

Listing of anatomical location, Talairach coordinates and maximum $z$ values for the activation-delay contrast of cue-target trials and no-cue-target trials. Cortical areas in the frontal cortex that show a delay in no-cue-target trials compared with cue-target trials with a minimum volume size of $200 \mathrm{~mm}^{3}$ and a $z$ value $>3.1$ are reported

\begin{tabular}{|c|c|c|c|c|}
\hline \multirow[t]{2}{*}{ Anatomical area } & \multicolumn{2}{|c|}{ Left hemisphere } & \multicolumn{2}{|c|}{ Right hemisphere } \\
\hline & $\begin{array}{l}\text { Talairach } \\
\text { coordinates }\end{array}$ & $Z_{\max }$ & $\begin{array}{l}\text { Talairach } \\
\text { coordinates }\end{array}$ & $Z_{\max }$ \\
\hline \multicolumn{5}{|l|}{ Frontal } \\
\hline IFJ (BA 6/9/44) & $-38,2,30$ & 6.648 & $46,11,30$ & 6.277 \\
\hline Middle frontal gyrus (BA 6) & $-29,-1,50$ & 4.407 & $23,2,47$ & 4.741 \\
\hline Medial frontal gyrus (BA 6) & & & $5,11,44$ & 5.525 \\
\hline Inferior frontal sulcus (BA 45/46) & $-31,41,15$ & 4.733 & $34,41,15$ & 5.564 \\
\hline Insula/inferior frontal gyrus (BA 44) & $-34,14,9$ & 5.044 & $31,17,15$ & 5.872 \\
\hline
\end{tabular}

dissociation. It might be that the left IFJ is more important for task preparation because of the verbal nature of the task.

\section{General Preparation versus Task Preparation}

The question of whether the cue-related activation reflects general preparation or task-specific preparation was clarified by the analysis of the time delay between cue-target trials and no-cue-target trials. While no difference in activation strength was found between these trial types, some cue-related frontal areas showed a significant time delay in no-cue-target trials compared with cue-target trials. Regarding the functional interpretation, this finding indicates that the cue-related frontal network is indeed cue-locked and not locked to the fixation cross that appeared at the beginning of each trial. This finding demonstrates that the frontal network identified in cue-only trials is task-preparation related. Furthermore, the omission of a difference in activation strength suggests that the taskpreparation process that takes place during a long cue-target interval was also involved when cue and target were presented concurrently. This has an important implication for the interpretation of the cue-related activation. It suggests that the reaction time difference of cue-target trials and no-cue-target trials does not reflect the operation of different processes but only a delaying of the preparation process when cue and target were presented simultaneously. This is crucial for the concept of task preparation and indicates that it is necessary whenever a task has to be implemented. With enough time, we can carry out this process in advance, otherwise we have to prepare immediately before task execution. 
This assumption was further supported by the analysis of the activation onset in cue-target trials with fast reaction times compared with cue-target trials with slow reaction times. Since subjects did not always prepare the task in cue-target trials, the task preparation process was delayed in some trials. This led to a slow reaction time and a delay of the frontal activation in these trials. Importantly, this time delay was not detected in the primary motor cortex, which would have been expected if it was primarily due to the delay of the motor response. It is probable that the time delay in the primary motor cortex is too small to be detected by the present methodology. Rather, a time delay was only located in anterior frontal regions that were related to task preparation. Hence, the activation onset in the frontal cortex predicts, to some degree, the size of the behavioral cueing effect in cue-target trials.

\section{Task Preparation in the IFJ and Pre-SMA}

The correlational analysis indicated that two frontal components play a major role in task preparation: the IFJ and pre-SMA. The IFJ is located at the border of premotor and prefrontal cortices and hence is anatomically perfectly suited for task management. It is widely accepted that the lateral premotor cortex is related to movement preparation and visuo-motor integration. However, recent neuroimaging research (Deiber et al., 1997), as well as research with non-human primates (Wise et al., 1996; Murray et $a l ., 2000)$, suggested that this not only holds true for direct stimulus-response mappings but also for so-called arbitrary or conditional motor mappings. In such mappings, a specific movement has to be selected in response to an arbitrary stimulus. This task requires the application of an abstract rule to guide behavior. Task preparation also requires the application of an abstract rule in the selection of the relevant stimulusresponse mappings. In this sense, task preparation might be an abstraction from arbitrary motor mapping. This interpretation is further supported by a recent study of Nagahama and colleagues in which they showed that two different prefrontal areas are related to task management (Nagahama et al., 2001). One was located in the anterior dorsal prefrontal cortex and was assumed to be related to 'higher order control of attention', while the other was located at the IFJ and was assumed to be related to 'the reorganization of stimulus response associations'. Even if the posterior prefrontal area in the study of Nagahama and colleagues (Nagahama et al., 2001) was located slightly more anterior than the activation we have found, the assumed process fits very well to what we have described as task preparation. In task preparation, subjects have to configure the associations between the stimuli and the task-relevant responses. Further support for this functional interpretation of the IFJ comes from a recent study by Konishi and colleagues, who identified the IFJ to be involved in transition between different task blocks (Konishi et al., 2001). On a less specific explanatory level, this assumption is also consistent with the idea that the lateral prefrontal cortex is involved in implementing control as proposed by MacDonald and colleagues (MacDonald et al., 2000).

The second frontal brain region that was significantly correlated with the behavioral cueing effect was the pre-SMA. This mesial frontal region is strongly connected with the lateral prefrontal cortex and the lateral premotor cortex. Previous research indicated that the pre-SMA is related to higher-order control and preparation of motor activity (Lee et al., 1999; Picard and Strick, 1996). It is very reasonable to assume that the pre-SMA and the IFJ have different functional roles in task preparation. While the fronto-lateral cortex might be involved in the selection of cue-related task rules, the pre-SMA might be responsible for implementing these rules on a higher-order level of motor control.

\section{Target-related Processing}

The contrast of cue-target trials and cue-only trials revealed the cortical areas that were related to target processing and response execution. Accordingly, activation was found in the premotor and primary motor cortex, reflecting the preparation and execution of the motor responses. Furthermore, a dissociation was found between target-related and cue-related processing in the fronto-median wall. While cue-related processing involved the pre-SMA, processing of the target was related to activation in the ACC. Previous studies showed that the ACC is involved in error detection, response inhibition and resolving response conflict (Cater et al., 2000; MacDonald et al., 2000; Ullsperger and von Cramon, 2001). In the present study, it is very reasonable to assume that the ACC is related to response conflict and response inhibition. Since targets were encoded in two different task sets, a response conflict might have been arisen when the target was encoded according to the irrelevant task set.

Furthermore, also the left IFJ showed target-related activation. As we have suggested, subjects did not always use the task cue; sometimes they waited until target presentation to start task preparation. Consequently, subtracting cue-only trials did not eliminate all preparation-related activation from cue-target trials.

\section{Task Preparation, Response Preparation and Task Switching}

The task-cue related network that was found to be activated in the present study overlaps to a high degree with results from response-cueing paradigms. It was found that the processing of spatial cues is related to a similar frontal network and was interpreted as reflecting spatial attentional mechanisms (Hopfinger $e t$ al., 2000). However, in the present task, it is not plausible to trace the cueing effect back to spatial attention, since the task did not involve any spatial processing.

Regarding the task-switching literature, the present findings clarify some controversial issues. The activated network is similar to the one found in a number of task-switching studies (Omori et al., 1999; Dove et al., 2000; Pollmann et al., 2000). However, in the present study this activation is not restricted to switch trials but involves repetition trials as well. This result is consistent with previous neuroimaging findings that the cortical areas which are activated in switch trials are also activated in repetition trials, when compared to a baseline (Dove et al., 2000). This might be due to the fact that in a paradigm in which switch and repetition trials are mixed within one experimental block, repetition trials also contain, to some extent, a 'switch' component. This assumption was supported by behavioral findings that the reaction time for repetition trials in blocks where only one task was presented (pure blocks) was faster than repetition trials in blocks where switch and repetition trials were mixed (as in the present experiment) (Kray and Lindenberger, 2000). This might explain why some studies, which used mixed blocks to compute switch-related activation, have found no frontal areas to be involved in task switching (Kimberg et al., 2000). Importantly, the present findings suggest that the frontal network is not involved in task switching per se but is related to task preparation.

\section{Conclusion}

The present findings revealed that a frontal network is involved in task preparation. Correlational analysis with the behavioral 
cueing effect suggest that the key components of this network are the left IFJ and the pre-SMA. We claim that the IFJ is responsible for implementing a task set, which requires the selection of the relevant stimulus-response mappings for the upcoming task. The pre-SMA might be responsible for implementing these task rules on a higher-order motor control level. Since task preparation is required as soon as more than one task set comes into play, it is not surprising that this network is involved in a number of different cognitive paradigms.

\section{Notes}

Address correspondence to Marcel Brass, Max Planck Institute of Cognitive Neuroscience, Stephanstrasse 1A, D-04103, Leipzig, Germany. Email: brass@cns.mpg.de.

\section{References}

Allport A, Styles EA, Hsieh S (1994) Shifting intentional set: exploring the dynamic control of tasks. In: Attention and performance XV (Umilta C, Moscovitch M, eds), pp. 421-452. Hillsdale, NJ: Erlbaum.

Bosch V (2000) Statistical analysis of multi-subject fMRI data: the assessment of focal activations. J Magn Reson Imaging 11:61-64.

Cater C, Macdonald AM, Botvinick M, Ross LL, Stenger A, Noll D, Cohen JD (2000) Parsing executive processes: strategic vs. evaluative functions of the anterior cingulate cortex. Proc Natl Acad Sci USA 97:1944-1948.

Deiber MP, Wise SP, Honda M, Catalan MJ, Grafman J, Hallett M (1997) Frontal and parietal networks of conditional motor learning: a positron emission tomography study. J Neurophysiol 78:977-991.

Dove A, Pollmann S, Schubert T, Wiggins CJ, von Cramon DY (2000) Prefrontal cortex activation in task switching: an event-related fMRI study. Cogn Brain Res 9:103-109.

Friston KJ, Holmes AP, Worsley KJ, Poline JP, Frith CD, Frackowiak RSJ (1995) Statistical parametric maps in functional imaging: a general linear approach. Hum Brain Mapp 2:189-210.

Friston KJ, Fletcher P, Josephs O, Holmes A, Rugg MD, Turner R (1998) Event-related fMRI: characterizing differential responses. Neuroimage 7:30-40.

Hopfinger JB, Buonocore MH, Mangun GR (2000) The neural mechanisms of top-down attentional control. Nat Neurosci 3:284-291.

Kimberg DY, Aguirre GK, D'Esposito M (2000) Modulation of task-related neural activity in task-switching: an fMRI study. Cogn Brain Res 10:189-196

Konishi S, Nakajima K, Uchida I, Kameyama M, Nakahara K, Sekihara K, Miyashita Y (1998) Transient activation of inferior prefrontal cortex during cognitive set shifting. Nat Neurosci 1:80-84.

Konishi S, Donaldson DI, Buckner RL (2001) Transient activation during block transition. Neuroimage 13:364-374.

Kray J, Lindenberger U (2000) Adult age differences in task switching. Psychol Aging 15:126-147.

Lee K-M, Chang K-H, Roh J-K (1999) Subregions within the supplementary motor area activated at different stages of movement preparation and execution. Neuroimage 9:117-123.

Lohmann G, Mueller K, Bosch V, Mentzel H, Hessler S, Chen L, Zysset S, von Cramon DY (2001) Lipsia - a new software system for the evaluation of functional magnetic resonance images of the human brain. Comput Med Imaging Graph 25:449-457.

MacDonald AW III, Cohen JD, Stenger VA, Carter CS (2000) Dissociating the role of the dorsolateral prefrontal and anterior cingulate cortex in cognitive control. Science 288:1835-1838.

Miezin FM, Maccotta L, Ollinger JM, Petersen SE, Buckner RL (2000) Characterizing the hemodynamic response: effects of presentation rate, sampling procedure, and the possibility of ordering brain activity based on relative timing. Neuroimage 11:735-759.

Meiran N (1996) Reconfiguration of processing mode prior to task performance. J Exp Psychol Learn Mem Cogn, 22:1423-1442.

Monsell S (1996) Control of mental processes. In: Mysteries of the mind: tutorial essays on cognition (Bruce V, ed.), pp. 93-148. Hove, UK: Erlbaum.

Murray EA, Bussey TJ, Wise SP (2000) Role of prefrontal cortex in a network for arbitrary visuomotor mapping. Exp Brain Res 133:114-129.

Nagahama Y, Okada T, Katsumi Y, Hayashi T, Yamauchi H, Oyanagi C, Konishi J, Fukuyama H, Shibasaki H (2001) Dissociable mechanisms of attentional control within the human prefrontal cortex. Cereb Cortex 11:85-92.

Oldfield RC (1971) The assessment and analysis of handedness: the Edinburgh Inventory. Neuropsychologia 9:97-113.

Omori M, Yamada H, Murata T, Sadato N, Tanaka M, Ishii Y, Isaki K, Yonekura Y (1999) Neuronal substrates participating in attentional set-shifting of rules for visually guided motor selection: a functional magnetic resonance imaging investigation. Neurosci Res 33:317-323.

Picard N, Strick PL (1996) Motor areas of the medial wall: a review of their location and functional activation. Cereb Cortex 6:342-353.

Pollmann S, Dove A, von Cramon DY, Wiggins CJ (2000) Event-related fMRI: comparison of conditions with varying BOLD overlap. Hum Brain Mapp 9:26-37.

Ratcliff R (1979) Group reaction time distributions and an analysis of distribution statistics. Psychol Bull 86:446-461.

Rogers RD, Monsell S (1995) Costs of a predictable switch between simple cognitive tasks. J Exp Psychol Gen 124:207-231.

Sohn M-H, Ursu S, Anderson JR, Stenger VA, Carter CS (2000) The role of prefrontal cortex and posterior parietal cortex in task switching. Proc Natl Acad Sci USA 97:13448-13453.

Sudevan P, Taylor DA (1987) The cueing and priming of cognitive operations. J Exp Psychol Hum Percept Perform 13:89-103.

Talairach P, Tournoux J (1988) A stereotactic coplanar atlas of the human brain. Stuttgart: Thieme.

Thirion JP (1998) Image matching as a diffusion process: an analogy with Maxwell's demons. Med Image Anal 2:243-260.

Ullsperger M, von Cramon DY (2001) Subprocesses of performance monitoring: a dissociation of error processing and response competition revealed by event-related fMRI and ERPs. Neuroimage 14:1387-1401.

Wise SP, di Pelligrino G, Boussaoud D (1996) The premotor cortex and nonstandard sensorimotor mapping. Can J Physiol Pharmacol 74:469-482. 